Francis Jordan, Department of Mathematics, West Virginia University, Morgantown, WV 26506-6310 e-mail: FJordan@math.wvu.edu

\title{
CARDINAL INVARIANTS CONNECTED WITH ADDING REAL FUNCTIONS
}

\begin{abstract}
In this paper we consider a cardinal invariant related to adding real functions defined on the real line. Let $\mathcal{F}$ be such a family, we consider the smallest cardinality of a family $\mathcal{G}$ of functions such that $h+\mathcal{G}$ has non-empty intersection with $\mathcal{F}$ for every function $h$. We note that this cardinal is the additivity, a cardinal previously studied, of the compliment of $\mathcal{F}$. Thus, we calculate the additivities of the compliments of various families of functions including the Darboux, almost continuous, extendable and perfect road functions. We briefly consider the general relationship between the additivity of a family and its compliment.
\end{abstract}

\section{Preliminaries}

In what follows we will use the following terminology and notation. Functions will be identified with their graphs. The set of all functions from a set $X$ into a set $Y$ will be denoted by $Y^{X}$. Given a set $X$ and $f, g \in X^{X}$ we denote their composition by $f \circ g$. The characteristic function of a set $A \subseteq \mathbb{R}$ will be denoted by $\chi_{A}$. The symbol $|X|$ will denote the cardinality of the set $X$. The successor of a cardinal $\kappa$ will be denoted by $\kappa^{+}$. We denote by $[X]^{<\kappa},[X]^{\kappa}$, and $[X] \leq \kappa$ the sets of all subsets of $X$ of cardinality less than $\kappa$, equal to $\kappa$, and less than or equal to $\kappa$, respectively. The cardinality of the real numbers $\mathbb{R}$ will be denoted by $\mathfrak{c}$. Given a cardinal number $\kappa$ we let $\operatorname{cf}(\kappa)$ denote the cofinality of $\kappa$. We say a cardinal $\kappa$ is regular provided that $\operatorname{cf}(\kappa)=\kappa$. For

Key Words: cardinal invariants; extendable, Darboux, almost continuous and peripherially continuous functions; functions with perfect road.

Mathematical Reviews subject classification: Primary: 26A15; Secondary: 03E75, $54 \mathrm{~A} 25$.

Received by the editors June 26, 1996

* This paper was written under supervision of K. Ciesielski. The author wishes to thank him for many helpful conversations. 
functions $f, g \in \mathbb{R}^{\mathbb{R}}$ let $[f=g]$ denote the set $\{x \in \mathbb{R}: f(x)=g(x)\}$. We define $[f<g]$ and $[f \leq g]$ in a similar way.

dense subset

We also will consider the following cardinal invariants related to a cardinal $\kappa$.

$$
\begin{aligned}
& d_{\kappa}=\min \left\{|F|: F \subseteq \kappa^{\kappa} \&\left(\forall g \in \kappa^{\kappa}\right)(\exists f \in F)(|[f=g]|=\kappa)\right\}, \\
& d_{\kappa}^{*}=\min \left\{|F|: F \subseteq \kappa^{\kappa} \&\left(\forall G \in\left[\kappa^{\kappa}\right]^{\kappa}\right)(\exists f \in F)(\forall g \in G)(|[f=g]|=\kappa\},\right. \\
& e_{\kappa}=\min \left\{|F|: F \subseteq \kappa^{\kappa} \&\left(\forall g \in \kappa^{\kappa}\right)(\exists f \in F)(|[f=g]|<\kappa)\right\}, \\
& e_{\kappa}^{*}=\min \left\{|F|: F \subseteq \kappa^{\kappa} \&\left(\forall G \in\left[\kappa^{\kappa}\right]^{\kappa}\right)(\exists f \in F)(\forall g \in G)(|[f=g]|<\kappa)\right\} .
\end{aligned}
$$

Note that $d_{\kappa} \leq d_{\kappa}^{*}$ and $e_{\kappa} \leq e_{\kappa}^{*}$.

\section{Introduction}

We will denote the complement of a family $\mathcal{F} \subseteq \mathbb{R}^{\mathbb{R}}$ by $\neg \mathcal{F}$. The following cardinal function has been defined for families $\mathcal{F} \subset \mathbb{R}^{\mathbb{R}}$ in [5].

$$
\left.\left.\mathrm{A}(\mathcal{F})=\min g \in \mathbb{R}^{\mathbb{R}}\right)(\exists f \in F)(f+g \in \neg \mathcal{F})\right\} \cup\left\{\left(2^{\mathfrak{c}}\right)^{+}\right\}
$$

If we restate the definition of additivity for the complement of a family $\mathcal{F}$ we get,

$$
\left.\left.\mathrm{A}(\neg \mathcal{F})=\min g \in \mathbb{R}^{\mathbb{R}}\right)(\exists f \in F)(f+g \in \mathcal{F})\right\} \cup\left\{\left(2^{\mathfrak{c}}\right)^{+}\right\}
$$

So, the statement $\mathrm{A}(\neg \mathcal{F}) \leq \kappa$ is equivalent to the fact that there is a family $F \subseteq \mathbb{R}^{\mathbb{R}}$ such that $|F|=\kappa$ and $(g+F) \cap \mathcal{F} \neq \emptyset$ for every $g \in \mathbb{R}^{\mathbb{R}}$.

Below we list some basic facts about this cardinal function [4].

Proposition 1. Let $\mathcal{G}, \mathcal{F} \subset \mathbb{R}^{\mathbb{R}}$. Then,

(i) $\mathrm{A}(\mathcal{F}) \geq 2$ if and only if $\mathcal{F} \neq \emptyset$;

(ii) $\mathrm{A}(\mathcal{F}) \leq 2^{\mathfrak{c}}$ if and only if $\mathcal{F} \neq \mathbb{R}^{\mathbb{R}}$;

(iii) if $\mathcal{F} \subset \mathcal{G}$, then $\mathrm{A}(\mathcal{F}) \leq \mathrm{A}(\mathcal{G})$;

(iv) if $\mathcal{F} \neq \emptyset$, then $\mathrm{A}(\mathcal{F})=2$ if and only if $\mathcal{F}-\mathcal{F} \neq \mathbb{R}^{\mathbb{R}}$.

Proof. Items (i), (ii), and (iii) are easy and are restatements of $[4$, Proposition 1.1].

Item (iv) is a modification of [4, Proposition 1.3] by replacing the assumption $\chi_{\emptyset} \in \mathcal{F}$ with $\mathcal{F} \neq \emptyset$. For the readers convenience we present here the modified proof. 
Suppose that $\mathcal{F}-\mathcal{F}=\mathbb{R}^{\mathbb{R}}$. We show that $\mathrm{A}(\mathcal{F})>2$. Let $f_{1}, f_{2} \in \mathbb{R}^{\mathbb{R}}$ be arbitrary and $F=\left\{f_{1}, f_{2}\right\}$. We find a $g \in \mathbb{R}^{\mathbb{R}}$ such that $g+f_{1}, g+f_{2} \in \mathcal{F}$. Since $\mathcal{F}-\mathcal{F}=\mathbb{R}^{\mathbb{R}}$, there exists $h_{1}, h_{2} \in \mathcal{F}$ such that $f_{1}-f_{2}=h_{1}-h_{2}$. Let $g=h_{1}-f_{1}=h_{2}-f_{2}$. Then $f_{i}+g=f_{i}+\left(h_{i}-f_{i}\right)=h_{i} \in \mathcal{F}$ for $i=1,2$. Thus, $\mathrm{A}(\mathcal{F})>2$.

To see the other implication suppose that $\mathcal{F}-\mathcal{F} \neq \mathbb{R}^{\mathbb{R}}$. Since $\mathcal{F} \neq \emptyset$, $\mathrm{A}(\mathcal{F}) \geq 2$, so it is enough to show that $\mathrm{A}(\mathcal{F}) \leq 2$. Pick $h \in \mathbb{R}^{\mathbb{R}} \backslash(\mathcal{F}-\mathcal{F})$ and put $F=\left\{\chi_{\emptyset}, h\right\}$. Let $g \in \mathbb{R}^{\mathbb{R}}$ be arbitrary. It is enough to show that $g+f \notin \mathcal{F}$ for some $f \in \mathcal{F}$. However, if $g=\chi_{\emptyset}+g \in \mathcal{F}$ and $h+g \in \mathcal{F}$, then $h \in \mathcal{F}-g \subseteq \mathcal{F}-\mathcal{F}$, contradicting our choice of $h$. Thus, $\mathrm{A}(\mathcal{F})=2$.

We prove some other facts about A with respect to the values it may assume and its relationship with complementation.

Proposition 2. Let $\omega \leq \lambda \leq 2^{\mathfrak{c}}$. There exists $\mathcal{F} \subseteq \mathbb{R}^{\mathbb{R}}$ such that $\mathrm{A}(\mathcal{F})=2$ and $\mathrm{A}(\neg \mathcal{F})=\lambda$.

Proof. Let $H$ be an additive subgroup of $\mathbb{R}^{\mathbb{R}}$ such that $|H|=\lambda$ and the set $\left\{g+H: g \in \mathbb{R}^{\mathbb{R}}\right\}=\left\{H_{\alpha}: \alpha \in 2^{\mathfrak{c}}\right\}$ has cardinality $2^{\mathfrak{c}}$. Let $\mathcal{F}$ be a selector of $\left\{H_{\alpha}: \alpha \in 2^{\mathfrak{c}}\right\}$.

We prove that $\mathrm{A}(\neg \mathcal{F})=\lambda$. We first show that $\mathrm{A}(\neg \mathcal{F}) \leq \lambda$. Since $|H|=\lambda$, it is enough to show that

$$
\left(\forall g \in \mathbb{R}^{\mathbb{R}}\right)(\exists h \in H)(g+h \in \mathcal{F}) .
$$

Let $g \in \mathbb{R}^{\mathbb{R}}$ be arbitrary. Clearly, $(g+H) \cap \mathcal{F} \neq \emptyset$. So, there exists an $h \in H$ such that $g+h \in \mathcal{F}$. Thus, (1) holds and $\mathrm{A}(\neg \mathcal{F}) \leq|H|=\lambda$.

Next we prove the other inequality. Let $F \subseteq \mathbb{R}^{\mathbb{R}}$ be such that $|F|=\kappa<\lambda$. We find a $g \in \mathbb{R}^{\mathbb{R}}$ such that $f+g \in \neg \mathcal{F}$ for all $f \in F$. Enumerate $F$ by $\left\{f_{\xi}: \xi<\kappa\right\}$. Each $f_{\xi}$ lies in some translation $H_{\alpha_{\xi}}$ of $H$. Since $\mathcal{F}$ is a selector of $\left\{H_{\alpha}: \alpha \in 2^{\mathfrak{c}}\right\}$,

there is a unique $h_{\xi} \in H$ such that $f_{\xi}+h_{\xi} \in H_{\alpha_{\xi}} \cap \mathcal{F}$.

Since $|F|=\kappa<\lambda$, there is an $h \in H \backslash\left\{h_{\xi}: \xi \in \kappa\right\}$. Thus, by the uniqueness part of $(2), h+f_{\xi} \in\left(H_{\alpha_{\xi}} \backslash \mathcal{F}\right) \subseteq \neg \mathcal{F}$ for every $\xi<\kappa$. So, letting $g=h$, we have $\lambda \leq \mathrm{A}(\neg \mathcal{F})$.

We show that $\mathrm{A}(\mathcal{F})=2$. Since $\mathcal{F} \neq \emptyset$, it is enough to show that $\mathrm{A}(\mathcal{F}) \leq 2$. Pick $h \in H \backslash\left\{\chi_{\emptyset}\right\}$. Let $F=\left\{\chi_{\emptyset}, h\right\}$ and $g \in \mathbb{R}^{\mathbb{R}}$ be arbitrary. It is enough to show that $g+f \notin \mathcal{F}$ for some $f \in \mathcal{F}$. If $g \notin \mathcal{F}$, then $g+\chi_{\emptyset} \notin \mathcal{F}$. On the other hand, if $g \in \mathcal{F}$, then $h+g \notin \mathcal{F}$ since $h \neq \chi_{\emptyset}$ and $\mathcal{F} \cap(g+H)=\{g\}$. So, for any $g \in \mathbb{R}^{\mathbb{R}}$ either $h+g \in \neg \mathcal{F}$ or $g+\chi_{\emptyset} \in \neg \mathcal{F}$. Thus $\mathrm{A}(\mathcal{F}) \leq 2$.

An immediate corollary of Proposition 2 is the following fact. 
Corollary 3. There exists families $\mathcal{G}, \mathcal{F} \subseteq \mathbb{R}^{\mathbb{R}}$ such that $\mathrm{A}(\mathcal{F})=\mathrm{A}(\mathcal{G})$ and $\mathrm{A}(\neg \mathcal{F}) \neq \mathrm{A}(\neg \mathcal{G})$.

Using a different argument we may extend Proposition 2 to include all finite cardinals.

Proposition 4. For every $2 \leq n \leq \omega$ there is an $\mathcal{F} \subseteq \mathbb{R}^{\mathbb{R}}$ such that $\mathrm{A}(\mathcal{F})=n$ and $\mathrm{A}(\neg \mathcal{F})=2$.

Proof. Let $S$ be the family of constant functions from $\mathbb{R}$ into $\mathbb{Z}$ and put $A_{k}=\{f \in S: f(0)=k$ modulo $\left.n)\right\}$ for every $0 \leq k \leq n-1$. Let $T$ be a selector of $\left\{h+S: h \in \mathbb{R}^{\mathbb{R}}\right\}$. Put $\mathcal{F}=\bigcup\left\{T+A_{k}: 1 \leq k \leq n-1\right\}$ and note that $\neg \mathcal{F}=T+A_{0}$.

We prove that $\mathrm{A}(\mathcal{F})=n$. We first show that $\mathrm{A}(\mathcal{F}) \geq n$. Let $F=$ $\left\{f_{0}, \ldots, f_{n-2}\right\} \subseteq \mathbb{R}^{\mathbb{R}}$ be arbitrary. We find a $g$ such that $g+F \subseteq \mathcal{F}$. For every $0 \leq t \leq n-2$ there is a $s_{t} \in S$ such that $f_{t} \in s_{t}+T$. Since $\left|\left\{A_{0}, \ldots, A_{n-1}\right\}\right|=n$ and $\left|\left\{s_{0}, \ldots, s_{n-2}\right\}\right|=n-1$, there is a $k^{*} \in\{0, \ldots, n-1\}$ such that $s_{t} \notin A_{k^{*}}$ for every $0 \leq t \leq n-2$. Let $s \in A_{k^{*}}$. Then $-s+s_{t} \notin A_{0}$ for all $0 \leq t \leq n-2$. Thus, putting $g=-s$, we have $g+f_{t} \in\left(-s+s_{t}\right)+T \subseteq \mathcal{F}$ for every $f_{t} \in F$. So, $\mathrm{A}(\mathcal{F}) \geq n$. We now show that $\mathrm{A}(\mathcal{F}) \leq n$. Let $F=\left\{f_{0}, \ldots, f_{n-1}\right\}$ where $f_{k} \in A_{k}$ for all $0 \leq k \leq n-1$. It is enough to show that

$$
\left(\forall g \in \mathbb{R}^{\mathbb{R}}\right)(\exists f \in F)(g+f \notin \mathcal{F}) .
$$

Let $g \in \mathbb{R}^{\mathbb{R}}$ be arbitrary. Since $F \subseteq S$, there is a $t \in T$ such that $g+F \subseteq t+S$. By way of contradiction assume that $g+F \subseteq \mathcal{F}$. Since $|g+F|=n$ and $\left|\left\{A_{0}, \ldots, A_{n-1}\right\}\right|=n$ and $(g+F) \cap\left(t+A_{0}\right)=\bar{\emptyset}$, there exist $0 \leq i<j \leq n-1$ such that $g+f_{i}, g+f_{j} \in t+A_{k}$ for some $1 \leq k \leq n-1$. So, there exist $s_{i}, s_{j} \in A_{k}$ such that $g+f_{i}=s_{i}+t$ and $g+f_{j}=s_{j}+t$. Then, $f_{i}-f_{j}=$ $\left(g+f_{i}\right)-\left(g+f_{j}\right)=\left(s_{i}+t\right)-\left(s_{j}+t\right)=s_{i}-s_{j} \in A_{0}$. It follows that there is some $1 \leq k \leq n-1$ such that $f_{i}, f_{j} \in A_{k}$ which contradicts our choice of $F$. Thus, $F$ satisfies $(3)$ and $\mathrm{A}(\mathcal{F}) \leq n$.

Since $\neg \mathcal{F} \neq \emptyset$, by Proposition 1 it is enough to show that $\mathrm{A}(\neg \mathcal{F}) \leq 2$. Let $h_{0}=\chi_{\emptyset} \in A_{0}$ and $h_{1} \in A_{1}$. We show that $\left\{h_{0}, h_{1}\right\}$ witnesses $\mathrm{A}(\mathcal{F}) \leq 2$, i.e,

$$
g+h_{0} \in \mathcal{F} \text { or } g+h_{1} \in \mathcal{F} \text { for all } g \in \mathbb{R}^{\mathbb{R}} .
$$

Let $g \in \mathbb{R}^{\mathbb{R}}$ be arbitrary. Since $h_{0}, h_{1} \in S$, there exists a $t \in T$ such that $g+h_{0}, g+h_{1} \in(t+S)$. In particular, there exists $s_{0}, s_{1} \in S$ such that $g+h_{0}=t+s_{0}$ and $g+h_{1}=t+s_{1}$. Since $s_{1}-s_{0}=\left(g+h_{1}\right)-\left(g+h_{0}\right)=h_{1} \in A_{1}$, clearly $s_{0}$ or $s_{1}$ is not in $A_{0}$. Thus, $\left\{h_{0}, h_{1}\right\}$ satisfies $(4)$. So $\mathrm{A}(\mathcal{F}) \leq 2$. 


\section{The Results}

We will primarily be concerned with calculating the additivities of the following families of functions from $\mathbb{R}$ into $\mathbb{R}$ and their complements. Some combinatorial characterizations of these cardinals are also given. We give general descriptions of these families that will work for any function from one space to another where the spaces are assumed to have the appropriate structure.

Dar: $f \in Y^{X}$ is a Darboux function if and only if $f[C]$ is connected in $Y$ for any connected subset $C$ of $X$.

Con: $f \in Y^{X}$ is a connectivity function if and only if the graph of $f$ restricted to $C$ is connected in $X \times Y$ for every connected subset $C$ of $X$.

AC: $f \in Y^{X}$ is an almost continuous function if and only if every open set in $X \times Y$ containing $f$ also contains some continuous function $g \in Y^{X}$.

EXT: $f \in Y^{X}$ is an extendable function if and only if there is a connectivity function $g: X \times[0,1] \rightarrow Y$ such that $f(x)=g(0, x)$ for every $x \in X$.

PR: $\quad f \in \mathbb{R}^{\mathbb{R}}$ is a perfect road function if and only if for every $x \in \mathbb{R}$ there is a perfect set $P \subset \mathbb{R}$ such that $x$ is a bilateral limit point of $P$ and $\left.f\right|_{P}$ is continuous at $x$.

PC: $f \in Y^{X}$ is a peripherally continuous function if and only if for every $x \in X$ and pair of open sets $U \subset X$ and $V \subset Y$ such that $x \in U$ and $f(x) \in V$ there is an open neighborhood $W$ of $x$ with $\operatorname{cl}(W) \subset U$ and $f[\operatorname{bd}(W)] \subset V$, where $\operatorname{cl}(W)$ and $\operatorname{bd}(W)$ denote the boundary and the closure of $W$, respectively.

SZ: $f \in Y^{X}$ is a Sierpiński-Zygmund function if and only if $f$ is continuous on no set of cardinality $\mathfrak{c}$.

The diagrams below describe the relations between the above families in $\mathbb{R}^{\mathbb{R}}$ except SZ. The symbol $\longrightarrow$ denotes containment. All inclusions are proper.

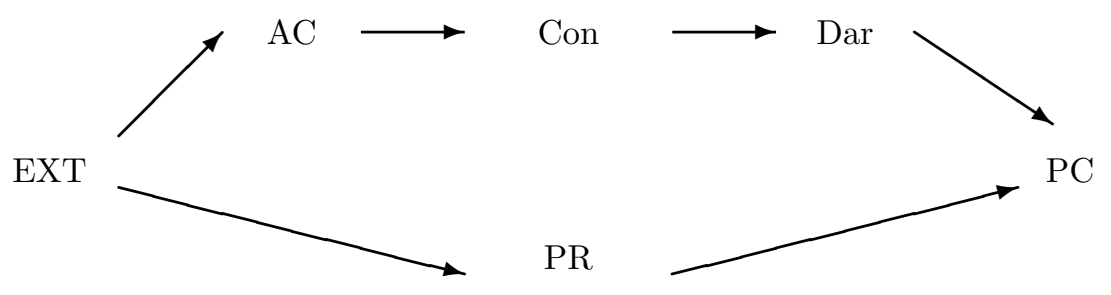


We will now discuss what may be said about the additivities of the families above and their respective complements in $\mathbb{R}^{\mathbb{R}}$. The additivities of the families EXT, PR, PC, Dar, Con, AC and SZ are known, as stated below.

Proposition 5. (i) (Ciesielski, Recław [4]) $\mathrm{A}(\mathrm{EXT})=\mathrm{A}(\mathrm{PR})=\mathfrak{c}^{+}$and $\mathrm{A}(\mathrm{PC})=2^{\mathfrak{c}}$

(ii) (Ciesielski, Miller [2]) $\mathrm{A}($ Dar $)=\mathrm{A}($ Con $)=\mathrm{A}(\mathrm{AC})=e_{\mathfrak{c}}$;

(iii) (Ciesielski, Natkaniec [3]) $\mathrm{A}(\mathrm{SZ})=d_{\mathfrak{c}}$.

We first calculate the numbers $\mathrm{A}(\neg \mathrm{EXT}), \mathrm{A}(\neg \mathrm{PR})$ and $\mathrm{A}(\neg \mathrm{PC})$, as stated in the two theorems below.

Theorem 6. $\mathrm{A}(\neg \mathrm{PR})=\mathrm{A}(\neg \mathrm{EXT})=2^{\mathfrak{c}}$.

Theorem 7. (Ciesielski [1]) $\mathrm{A}(\neg \mathrm{PC})=\omega_{1}$.

Next, we prove two theorems relating the additivies of $\neg$ Dar, $\neg$ Con, $\neg \mathrm{AC}$, and $\neg \mathrm{SZ}$ to some cardinal invariants of a combinatorial nature.

Theorem 8. $\mathrm{A}(\mathrm{SZ})=d_{\mathfrak{c}} \leq \mathrm{A}(\neg \mathrm{Dar}) \leq \mathrm{A}(\neg \mathrm{Con}) \leq \mathrm{A}(\neg \mathrm{AC}) \leq d_{\mathfrak{c}}^{*}$.

Theorem 9. $\mathrm{A}(\mathrm{AC})=\mathrm{A}(\mathrm{Con})=\mathrm{A}($ Dar $)=e_{\mathfrak{c}} \leq \mathrm{A}(\neg \mathrm{SZ}) \leq e_{\mathfrak{c}}^{*}$.

We then have two purely combinatorial theorems. The first will allow us to replace the inequalities of Theorems 8 and 9 with equalities under certain assumptions. The second will allow us to relate the additivies of some of these families with respect to complementation, e.g. $\mathrm{A}(\neg \mathrm{Dar}) \leq \mathrm{A}(\mathrm{Dar})$, under certain assumptions. The first part of Theorem 10 is due to Ciesielski [1].

Theorem 10. If $\left|[\mathfrak{c}]^{<\mathfrak{c}}\right|=\mathfrak{c}$, then $d_{\mathfrak{c}}=d_{\mathfrak{c}}^{*}$ and $e_{\mathfrak{c}}=e_{\mathfrak{c}}^{*}$.

Theorem 11. If $\left|[\mathfrak{c}]^{<\mathfrak{c}}\right|=\mathfrak{c}$ and $\mathfrak{c}=\lambda^{+}$, then $d_{\mathfrak{c}} \leq e_{\mathfrak{c}}$.

Theorem 8 and the first part of Theorem 10 yield:

Corollary 12. If $\left|[\mathfrak{c}]^{<\mathfrak{c}}\right|=\mathfrak{c}$, then

$\left.\mathrm{A}(\mathrm{SZ})=d_{\mathfrak{c}}=\mathrm{A}(\neg \mathrm{Dar})=\mathrm{A}(\neg \mathrm{Con})=\mathrm{A}(\neg \mathrm{AC})\right)=d_{\mathfrak{c}}^{*}$.

Theorem 9 and the second part of Theorem 10 imply:

Corollary 13. If $\left|[\mathfrak{c}]^{<\mathfrak{c}}\right|=\mathfrak{c}$, then

$\mathrm{A}($ Dar $)=\mathrm{A}($ Con $)=\mathrm{A}(\mathrm{AC})=e_{\mathfrak{c}}=\mathrm{A}(\neg \mathrm{SZ})=e_{\mathfrak{c}}^{*}$. 
From Theorem 11 and Corollaries 12 and 13 we can also conclude that:

Corollary 14. If $\left|[\mathfrak{c}]^{<\mathfrak{c}}\right|=\mathfrak{c}$ and $\mathfrak{c}=\lambda^{+}$, then

$\mathrm{A}(\mathrm{SZ})=\mathrm{A}(\neg \mathrm{Dar})=\mathrm{A}(\neg \mathrm{AC})=d_{\mathfrak{c}} \leq e_{\mathfrak{c}}=\mathrm{A}(\mathrm{Dar})=\mathrm{A}(\mathrm{AC})=\mathrm{A}(\neg \mathrm{SZ})$.

Finally, we quote two consistency results.

Proposition 15. (Ciesielski, Natkaniec [3]) Let $\lambda \geq \kappa \geq \omega_{2}$ be cardinals such that $\operatorname{cf}(\lambda)>\omega_{1}$ and $\kappa$ is regular. Then it is relatively consistent with $\mathrm{ZFC}+\mathrm{CH}$ that $2^{\mathfrak{c}}=\lambda$ and $\mathrm{A}(\mathrm{Dar})=\mathrm{A}(\mathrm{SZ})=\kappa$.

Proposition 16. (Ciesielski, Natkaniec [3]) Let $\lambda>\omega_{2}$ be a cardinal such that $\operatorname{cf}(\lambda)>\omega_{1}$. Then it is relatively consistent with $Z F C+C H$ that $2^{\mathfrak{c}}=\lambda$, and $\mathrm{A}(\mathrm{SZ})=\mathfrak{c}^{+}<2^{\mathfrak{c}}=\mathrm{A}($ Dar $)$.

Since, CH implies that $\left|\left[\mathfrak{c}^{<\mathfrak{c}}\right]\right|=\mathfrak{c}$, Propositions 15 and 16 together with Corollaries 12 and 13 imply immediately the following two corollaries.

Corollary 17. Let $\lambda \geq \kappa \geq \omega_{2}$ be cardinals such that $\operatorname{cf}(\lambda)>\omega_{1}$ and $\kappa$ is regular. Then it is relatively consistent with $Z F C+C H$ that $2^{\mathfrak{c}}=\lambda$ and

$\mathrm{A}(\mathrm{AC})=\mathrm{A}(\mathrm{Dar})=\mathrm{A}(\mathrm{SZ})=\mathrm{A}(\neg(\mathrm{Dar})=\mathrm{A}(\neg \mathrm{AC})=\kappa$.

Corollary 18. Let $\lambda>\omega_{2}$ be a cardinal such that $\operatorname{cf}(\lambda)>\omega_{1}$. Then it is relatively consistent with $\mathrm{ZFC}+\mathrm{CH}$ that $2^{\mathfrak{c}}=\lambda$, and

$\mathrm{A}(\neg$ Dar $)=\mathrm{A}(\neg \mathrm{AC})=\mathrm{A}(\mathrm{SZ})=\mathfrak{c}^{+}<2^{\mathfrak{c}}=\mathrm{A}(\mathrm{Dar})=\mathrm{A}(\mathrm{AC})=\mathrm{A}(\neg \mathrm{SZ})$.

The importance of the assumptions in Theorems 10 and 11 is not clear. In particular, the following problem is still open.

Problem 1. When does either $d_{\mathfrak{c}}=d_{\mathfrak{c}}^{*}$ or $e_{\mathfrak{c}}=e_{\mathfrak{c}}^{*}$ hold?

\section{Families EXT, PR, and PC}

To prove Theorem 6 we will use the following lemma which can be found in [4, Lemma 2.2].

Lemma 19. If $B \subset \mathbb{R}$ has cardinality $\mathfrak{c}, H \subset \mathbb{Q}^{B}$, and $|H|<2^{\mathfrak{c}}$, then there is a $g \in \mathbb{Q}^{B}$ such that $h \bigcap g \neq \emptyset$ for every $h \in H$. 
It may be of interest to notice that Lemma 19 is used in [4] to prove that $\mathrm{A}(\mathrm{PC})=2^{\mathfrak{c}}$.

Lemma 20. Let $A \subset \mathbb{R},|A|=\mathfrak{c}$, and

$$
\kappa=\min \left\{|F|: F \subset \mathbb{R}^{A} \&\left(\forall g \in \mathbb{R}^{A}\right)(\exists f \in F)\left(g+f \in \mathbb{R}^{A} \text { is bounded }\right)\right\} .
$$

Then $\kappa=2^{\mathfrak{c}}$.

Proof. It is enough to show that $2^{\mathfrak{c}} \leq \kappa$. Let $F \in\left[\mathbb{R}^{A}\right]^{<2^{\mathfrak{c}}}$. We will find $g \in \mathbb{R}^{A}$ such that $f+g$ is not bounded for every $f \in F$. Let $\left\{B_{n}: n \in \omega\right\}$ be a partition of $A$ such that $\left|B_{n}\right|=\mathfrak{c}$ for all $n \in \omega$. Fix $n \in \omega$. For each $f \in F$ choose $h_{n}^{f}: B_{n} \rightarrow \mathbb{Q}$ such that

$$
f(x)+h_{n}^{f}(x)>n \text { for every } x \in B_{n} .
$$

Now by Lemma 19 used with the sets $B_{n}$ and $\left\{h_{n}^{f}: f \in F\right\}$, there is a $g_{n}: B_{n} \rightarrow$ $\mathbb{Q}$ such that,

$$
(\forall f \in F)\left(\exists x \in B_{n}\right)\left(h_{n}^{f}(x)=g_{n}(x)\right) .
$$

Let $g=\bigcup\left\{g_{n}: n \in \omega\right\}$. Then, by (5) and (6), for every $n \in \omega$ and $f \in F$ there exists $x \in B_{n} \subset A$ such that $f(x)+g_{n}(x)=f(x)+g(x)>n$. So, $f+g$ is unbounded for every $f \in F$. Thus, $2^{\mathfrak{c}} \leq \kappa$, and the proof is complete.

Proof of Theorem 6. Notice that, by Proposition 1 , to prove Theorem 6 it is enough to show that $\mathrm{A}(\neg \mathrm{PR}) \geq 2^{\mathfrak{c}}$ since $\neg \mathrm{PR} \subset \neg \mathrm{EXT}$ and $\neg \mathrm{EXT} \neq \mathbb{R}^{\mathbb{R}}$.

Let $F \subset \mathbb{R}^{\mathbb{R}}$ and $|F|<2^{\mathfrak{c}}$. Choose a partition $\left\{B_{\alpha}: \alpha \in \mathfrak{c}\right\}$ of $\mathbb{R}$ into disjoint Bernstein sets, let $\left\{P_{\alpha}: \alpha<\mathfrak{c}\right\}$ be an enumeration of the perfect sets in $\mathbb{R}$, and put $I_{\alpha}=P_{\alpha} \cap B_{\alpha}$. Note that $\left|I_{\alpha}\right|=\mathfrak{c}$ and $I_{\alpha} \cap I_{\beta}=\emptyset$ for $\alpha<\beta<\mathfrak{c}$. Fix $\alpha<\mathfrak{c}$ and let $F_{\alpha}=\left\{\left.f\right|_{I_{\alpha}}: f \in F\right\}$. By Lemma 20 there is some $g_{\alpha}: I_{\alpha} \rightarrow \mathbb{R}$ such that $g_{\alpha}+\left.f\right|_{I_{\alpha}}$ is not bounded for every $\left.f\right|_{I_{\alpha}} \in F_{\alpha}$. Let $g \in \mathbb{R}^{\mathbb{R}}$ extend $\bigcup\left\{g_{\alpha}: \alpha<\mathfrak{c}\right\}$. Clearly $f+g$ is not bounded on any perfect set for every $f \in F$. In particular, $f+g$ is nowhere continuous on every perfect set, so $f+g \notin \mathrm{PR}$ for every $f \in F$. Therefore $\mathrm{A}(\neg \mathrm{PR})=2^{\mathfrak{c}}$.

Theorem 7 is a corollary of the following lemma.

Lemma 21. There exists a family $\mathcal{F}=\left\{f_{\xi} \in \mathbb{R}^{\mathbb{R}}: \xi \in \omega_{1}\right\}$ with the property that for every $g \in \mathbb{R}^{\mathbb{R}}$ there exists $\xi<\omega_{1}$, such that $f_{\xi}+g$ is dense in $\mathbb{R}^{2}$.

Proof. Let $\left\{h_{\alpha}: \alpha \in \mathfrak{c}\right\}=\left\{h \in \omega_{1}^{\mathbb{Q}}: h\right.$ is injective $\}$ and let $\left\{B_{\alpha}: \alpha<\mathfrak{c}\right\}$ be a partition of $\mathbb{R}$ with each $B_{\alpha}$ dense in $\mathbb{R}$. For each $\alpha<\mathfrak{c}, x \in B_{\alpha}$, and $\xi<\omega_{1}$ let $f_{\xi}(x)=h_{\alpha}^{-1}(\xi)$ if $\xi \in h_{\alpha}[\mathbb{Q}]$, otherwise let $f_{\xi}(x)$ be arbitrary. We show $\mathcal{F}$ has the desired property. 
Let

$$
\mathcal{Y}=\{\langle p, q\rangle \times\langle r, s\rangle: p, q, r, s \in \mathbb{Q}, p<q, r<s\}=\left\{I^{n} \times J^{n}: n<\omega\right\} .
$$

By way of contradiction assume there is some $g \in \mathbb{R}^{\mathbb{R}}$ such that $f_{\xi}+g$ is not dense in $\mathbb{R}^{2}$ for every $\xi<\omega_{1}$. Then for every $\xi<\omega_{1}$ there exist $I_{\xi} \times J_{\xi} \in \mathcal{Y}$ such that,

$$
\left(f_{\xi}+g\right) \cap\left(I_{\xi} \times J_{\xi}\right)=\emptyset .
$$

So there is some $n \in \omega$ such that the set $S=\left\{\xi \in \omega_{1}: I_{\xi} \times J_{\xi}=I^{n} \times J^{n}\right\}$ is uncountable. Let $S_{0}$ be a countable subset of $S$ and let $h: \mathbb{Q} \rightarrow S_{0}$ be a bijection. Then there is an $\alpha<\mathfrak{c}$ such that $h=h_{\alpha}$. Pick $x \in B_{\alpha} \cap I_{n}$. Then, by the definition of $f_{\xi}$, we have

$$
\left\{f_{\xi}(x)+g(x): \xi \in S_{0}\right\}=\left\{f_{\xi}(x): \xi \in h_{\alpha}[\mathbb{Q}]\right\}+g(x)=\mathbb{Q}+g(x) .
$$

Since $\mathbb{Q}+g(x)$ is dense in $\mathbb{R}$, there exists $y \in(\mathbb{Q}+g(x)) \cap J^{n}$. Therefore, $\left\langle x,\left(f_{\xi}+g\right)(x)\right\rangle \in I^{n} \times J^{n}=I_{\xi} \times J_{\xi}$, which contradicts $(7)$.

Proof of Theorem 7. It is easy to see that if $f \in \mathbb{R}^{\mathbb{R}}$ is dense in the plane, then $f \in$ PC. So by Lemma 21 there is a family $\mathcal{F} \in \mathbb{R}^{\mathbb{R}}$ of cardinality $\omega_{1}$ such that

$$
\left(\forall g \in \mathbb{R}^{\mathbb{R}}\right)(\exists f \in \mathcal{F})(g+f \in \mathrm{PC}) .
$$

Thus, $\mathrm{A}(\neg \mathrm{PC}) \leq \omega_{1}$.

To see the other inequality let $\left\{f_{n}: n \in \omega\right\}$ be a countable family of functions in $\mathbb{R}^{\mathbb{R}}$. It is enough to find a $g \in \mathbb{R}^{\mathbb{R}}$ such that $f_{n}+g \notin \mathrm{PC}$ for every $n \in \omega$. Define $g$ so that, $\left|\left(f_{n}+g\right)[(n, n+1)] \cap(0,1)\right|=1$ for every $n \in \omega$ and extend $g$ arbitrarily. Clearly $g$ is as desired.

\section{Families Dar, Con, and AC}

In this section we prove Theorem 8. By containment, we immediately have $\mathrm{A}(\neg \mathrm{Dar}) \leq \mathrm{A}(\neg \mathrm{Con}) \leq \mathrm{A}(\neg \mathrm{AC})$. Thus, it is enough to prove the inequalities $d_{\mathfrak{c}} \leq \mathrm{A}(\neg$ Dar $)$ and $\mathrm{A}(\neg \mathrm{AC}) \leq d_{\mathfrak{c}}^{*}$.

For the proof of $d_{\mathfrak{c}} \leq \mathrm{A}(\neg$ Dar $)$ we need the following definitions. For $A \subseteq \mathbb{R}$ let $\operatorname{LIN}(A)$ denote the linear subspace of $\mathbb{R}$ over $\mathbb{Q}$ spanned by $A$. Given a linear subspace $S$ of $\mathbb{R}$ over $\mathbb{Q}$ we call any set of the form $t+S$, with $t \in \mathbb{R}$, a translation of $S$. Let $\operatorname{Dar}^{*} \subseteq \mathbb{R}^{\mathbb{R}}$ stand for the family of nowhere-constant Darboux functions and $\operatorname{Dar}(\mathfrak{c})$ for the family of functions $f \in \mathbb{R}^{\mathbb{R}}$ such that

$$
\left|f^{-1}(y) \cap(a, b)\right|=\mathfrak{c}
$$

for all $a, b, y \in \mathbb{R}^{\mathbb{R}}$, with $a<b$. In the proof we will also use the following lemmas. 
Lemma 22. Let $H$ be a linear base of $\mathbb{R}$ over $\mathbb{Q}$ and $H_{1} \subseteq H$ be such that $\left|H \backslash H_{1}\right|=\mathfrak{c}$. Then $\operatorname{LIN}\left(S \cup H_{1}\right) \neq \mathbb{R}$ for any linear subspace $S$ such that $|S|<\mathfrak{c}$

Proof. Let $H_{0}=H \backslash H_{1}$ and $N=\operatorname{LIN}\left(H_{1}\right)$. By the linear independence of $H$, we have that $\left\{h+N: h \in H_{0}\right\}$ is a collection of pairwise disjoint translations of $N$. In particular, $N$ has $\mathfrak{c}$-many pairwise disjoint translations. Since $|S|<\mathfrak{c}$, there is a translation $T$ of $N$ such that $(s+N) \cap T=\emptyset$ for every $s \in S$. Thus, $T \cap(\bigcup\{s+N: s \in S\})=\emptyset$. Since $\bigcup\{s+N: s \in S\}=\operatorname{LIN}\left(S \cup H_{1}\right)$, we have $\operatorname{LIN}\left(S \cup H_{1}\right) \neq \mathbb{R}$.

Lemma 23. Let $N$ be a linear subspace of $\mathbb{R}$ over $\mathbb{Q}$ with $|N|=\mathfrak{c}$. Then $|I \cap N|=\mathfrak{c}$ for every non-degenerate interval $I \subseteq \mathbb{R}$.

Proof. Let $H$ be a linear base for $N$ over $\mathbb{Q}$. Note that $|H|=\mathfrak{c}$. Since $H$ is linearly independent, $\{h \cdot(\mathbb{Q} \backslash\{0\}): h \in H\}$ is a collection of pairwise disjoint dense subsets of $\mathbb{R}$. Since $\bigcup\{h \cdot(\mathbb{Q} \backslash\{0\}): h \in H\} \subseteq N$, it follows that $|I \cap N|=\mathfrak{c}$ for all non-degenerate intervals $I \subseteq \mathbb{R}$.

Lemma 24. $\mathrm{A}(\neg$ Dar $)>\mathfrak{c}$.

Proof. Let $H$ be a linear base for $\mathbb{R}$ over $\mathbb{Q}$ and $H_{1} \subseteq H$ be such that $\left|H_{1}\right|=\left|H \backslash H_{1}\right|=\mathfrak{c}$. Let $F=\left\{f_{\alpha} \in \mathbb{R}^{\mathbb{R}}: \alpha<\mathfrak{c}\right\}$ be an arbitrary family of functions of cardinality $\mathfrak{c}$. It is enough to find a $g \in \mathbb{R}^{\mathbb{R}}$ such that $f+g \notin$ Dar for all $f \in F$.

Let $N=\operatorname{LIN}\left(H_{1}\right)$ and $P_{\alpha, x}=\left\{f_{\beta}(x): \beta \leq \alpha\right\}$ for every $\alpha<\mathfrak{c}$ and $x \in \mathbb{R}$. Let $\left\{x_{\alpha}: \alpha<\mathfrak{c}\right\}$ be an enumeration of $\mathbb{R}$. Define $g \in \mathbb{R}^{\mathbb{R}}$ so that

$$
g\left(x_{\alpha}\right) \in \mathbb{R} \backslash \operatorname{LIN}\left(N_{\alpha, x_{\alpha}} \cup N\right),
$$

where $N_{\alpha, x_{\alpha}}=\operatorname{LIN}\left(P_{\alpha, x_{\alpha}} \cup\left\{\left(f_{\alpha}-g\right)\left(x_{0}\right)\right\}\right)$.

We may make such a choice since $H_{1}$ and $S=N_{\alpha, x_{\alpha}}$ satisfy the hypothesis of Lemma 22. We show $g$ is the desired function. Fix $\alpha<\mathfrak{c}$. If $\alpha \leq \beta<\mathfrak{c}$, then, by (8), $g\left(x_{\beta}\right) \notin N_{\beta, x_{\beta}}$. Since $f_{\alpha}\left(x_{\beta}\right) \in N_{\beta, x_{\beta}}$ and $g\left(x_{\beta}\right) \notin \operatorname{LIN}\left(N_{\beta, x_{\beta}} \cup N\right)$, we have $f_{\alpha}\left(x_{\beta}\right)+g\left(x_{\beta}\right) \notin \operatorname{LIN}\left(N_{\beta, x_{\beta}} \cup N\right)$. In particular, $f_{\alpha}\left(x_{\beta}\right)+g\left(x_{\beta}\right) \notin N$ for all $\beta>\alpha$. Thus, $\left|\left(f_{\alpha}+g\right)[\mathbb{R}] \cap N\right|<\mathfrak{c}$. Since $|N|=\mathfrak{c}$, by Lemma 23, $|I \cap N|=\mathfrak{c}$ for every non-degenerate interval. It follows that the range of $f_{\alpha}+g$ contains no non-degenerate interval. But, by (8), $f_{\alpha}+g$ takes at least two values and so, it is not constant. Therefore, $g+f_{\alpha} \notin$ Dar which completes the proof, since $\alpha<\mathfrak{c}$ was arbitrary.

Lemma 25. $\mathrm{A}(\neg$ Dar $)=\mathrm{A}\left(\neg \operatorname{Dar}^{*}\right)$. 
Proof. Since $\neg$ Dar $\subseteq \neg$ Dar* ${ }^{*}$, the inequality $\mathrm{A}(\neg$ Dar $) \leq \mathrm{A}\left(\neg\right.$ Dar $\left.^{*}\right)$ is obvious. We show the other inequality.

Let $F \subseteq \mathbb{R}^{\mathbb{R}}$ be a family of cardinality $\kappa=\mathrm{A}(\neg$ Dar $)$ witnessing the definition of $\mathrm{A}(\neg \mathrm{Dar})$, i.e.,

$$
\left(\forall g \in \mathbb{R}^{\mathbb{R}}\right)(\exists f \in F)(f+g \in \text { Dar }) .
$$

It is enough to find a family $F^{*} \subseteq \mathbb{R}^{\mathbb{R}}$ of cardinality $\kappa$ such that,

$$
\left(\forall g \in \mathbb{R}^{\mathbb{R}}\right)\left(\exists f \in F^{*}\right)\left(f+g \in \operatorname{Dar}^{*}\right) .
$$

Let $\mathcal{I}$ be the family of collections of mutually disjoint non-degenerate open intervals. Since there are continuum many open intervals and the cardinality of any disjoint collection of open intervals is at most $\omega$, it follows that $|\mathcal{I}|=\mathfrak{c}$. For each $I \in \mathcal{I}$ pick $h_{I} \in \operatorname{Dar}(\mathfrak{c})$ such that $h_{I}(x)=0$ if $x$ is an endpoint of any $i \in I$. Let $k_{I}$ be defined by $k_{I}(x)=\chi_{\cup I}(x) h_{I}(x)$ for each $x \in \mathbb{R}$. Let $K=\left\{k_{I}: I \in \mathcal{I}\right\}$. Note that $|K|=\mathfrak{c}$. Define $F^{*}=\{f+h: f \in F \& h \in K\}$. Since $|K|=\mathfrak{c}$ and, by Lemma $24, \mathfrak{c}<\kappa$ we have $\left|F^{*}\right|=\kappa$. So it is enough to show that $F^{*}$ satisfies (10).

Let $g \in \mathbb{R}^{\mathbb{R}}$ be arbitrary. By (9), there exists an $f \in F$ such that $f+g \in$ Dar. The set of points at which $f+g$ is constant form a countable collection $J$ of mutually disjoint non-degenerate open intervals such that $f+g$ is constant on each $j \in J$ and is nowhere-constant on $\mathbb{R} \backslash \bigcup J$. Since $\left(f+k_{J}\right) \in F^{*}$, it is enough to show that $\left(f+k_{J}\right)+g \in$ Dar* $^{*}$. We first show that $\left(f+k_{J}\right)+g$ is nowhere-constant. Let $x \in \mathbb{R}$ be arbitrary. If $x \in \operatorname{cl}(\bigcup J)$, then any open neighborhood $U$ about $x$ contains a non-degenerate sub-interval $i$ of some $j \in J$. Thus,

$$
\left((f+g)+k_{J}\right)[U] \supseteq\left((f+g)+k_{J}\right)[i]=\{r\}+k_{J}[i]=\{r\}+\mathbb{R}=\mathbb{R},
$$

where $\{r\}=(f+g)[j]$. So $\left(f+k_{J}\right)+g$ is not locally constant at $x$. If $x \notin \operatorname{cl}(\bigcup J)$, then there is a neighborhood $U \subseteq \mathbb{R} \backslash \operatorname{cl}(\bigcup J)$ of $x$ such that $k_{J}$ is equal to 0 on $U$, and $\left.\left(f+k_{J}+g\right)\right|_{U}=\left.(f+g)\right|_{U}$ which is non-constant on $U$. So, $f+g$ is non-constant at $x$. Thus, $\left(f+k_{J}\right)+g$ is nowhere-constant.

We now must show that $\left(f+k_{J}\right)+g$ is Darboux. Let $i \subseteq \mathbb{R}$ be a nondegenerate open interval. If $i \cap j \neq \emptyset$ for some $j \in J$, then $i$ contains a nontrivial sub-interval of $j$, so, arguing as in $(11),\left(\left(f+k_{J}\right)+g\right)[i]=\mathbb{R}$. If $i \cap j=\emptyset$ for all $j \in J$, then $\left(\left(f+k_{J}\right)+g\right)[i]=(f+g)[i]$. In either case $\left(\left(f+k_{J}\right)+g\right)[i]$ is an interval. Thus, $\left(f+k_{J}\right)+g$ is Darboux. So, $\left(f+k_{J}\right)+g \in$ Dar* $^{*}$ and $F^{*}$ satisfies (10) completing the proof.

Lemma 26. There exists an additive function $\Theta \in \mathbb{R}^{\mathbb{R}}$ such that $\Theta \circ f \in \operatorname{Dar}(\mathfrak{c})$ for every $f \in$ Dar*. 
Proof. Let $\Theta \in \operatorname{Dar}(\mathfrak{c})$ be an additive function. Such a function may be constructed as follows. Pick a linear base $H$ for $\mathbb{R}$ over $\mathbb{Q}$. Partition $H$ into two sets $H_{1}$ and $H_{2}$ of size $\mathfrak{c}$ and define $f: H \rightarrow \mathbb{R}$ so that $f\left[H_{1}\right]=0$ and $f\left[H_{2}\right]=\mathbb{R}$. The unique additive extension $\Theta$ of $f$ belongs to $\operatorname{Dar}(\mathfrak{c})$.

We show that $\Theta$ has the desired property. Let $f \in \operatorname{Dar}^{*}$ be arbitrary. For any $a<b$ and $y \in \mathbb{R}$, we have,

$$
\begin{aligned}
\left|(a, b) \bigcap(\Theta \circ f)^{-1}(y)\right| & =|\{x \in(a, b): \Theta(f(x))=y\}| \\
& \geq|\{z \in f[(a, b)]: \Theta(z)=y\}| \\
& =\mathfrak{c} .
\end{aligned}
$$

The last equality follows from the facts that $f[(a, b)]$ is a non-degenerate interval since $f \in \operatorname{Dar}^{*}$ and that $\Theta \in \operatorname{Dar}(\mathfrak{c})$.

Lemma 27. $\mathrm{A}(\neg \operatorname{Dar})=\mathrm{A}(\neg \operatorname{Dar}(\mathfrak{c}))$.

Proof. Since $\neg$ Dar $\subseteq \neg \operatorname{Dar}(\mathfrak{c})$, we have $\mathrm{A}(\neg \operatorname{Dar}) \leq \mathrm{A}(\neg \operatorname{Dar}(\mathfrak{c}))$. We show the other inequality.

Let $F \subseteq \mathbb{R}^{\mathbb{R}}$ have cardinality $\kappa=\mathrm{A}(\neg$ Dar $)$. We may assume, by Lemma 25 , that $F$ witnesses the definition of $\mathrm{A}\left(\neg \mathrm{Dar}^{*}\right)$, i.e.,

$$
\left(\forall g \in \mathbb{R}^{\mathbb{R}}\right)(\exists f \in F)\left(f+g \in \operatorname{Dar}^{*}\right) .
$$

It is enough to find a family $F^{*}$ of cardinality at most $\kappa$ such that $F^{*}$ witnesses the definition of $\mathrm{A}(\neg \operatorname{Dar}(\mathfrak{c}))$, i.e.,

$$
\left(\forall g \in \mathbb{R}^{\mathbb{R}}\right)\left(\exists f^{*} \in F^{*}\right)(f+g \in \operatorname{Dar}(\mathfrak{c})) .
$$

Define $F^{*}=\{\Theta \circ f: f \in F\}$ where $\Theta$ is the additive function from Lemma 26. Clearly $\left|F^{*}\right| \leq \kappa$. It is enough to show that $F^{*}$ satisfies (13). Let $g \in \mathbb{R}^{\mathbb{R}}$ be arbitrary. Pick $g_{1} \in \mathbb{R}^{\mathbb{R}}$ so that $\Theta \circ g_{1}=g$. By (12) there is an $f \in F$ such that $f+g_{1} \in$ Dar* $^{*}$. By our choice of $\Theta$, we have

$$
(\Theta \circ f)+g=\Theta \circ f+\Theta \circ g_{1}=\Theta \circ\left(f+g_{1}\right) \in \operatorname{Dar}(\mathfrak{c}) .
$$

Thus, $F^{*}$ satisfies (13), completing the proof.

Proof of $d_{\mathfrak{c}} \leq \mathrm{A}(\neg$ Dar $)$. Since $\mathrm{A}(\neg$ Dar $)=\mathrm{A}(\neg \operatorname{Dar}(\mathfrak{c}))$, it is enough for us to show that $d_{\mathfrak{c}} \leq \mathrm{A}(\neg \operatorname{Dar}(\mathfrak{c}))$. Let $\kappa=d_{\mathfrak{c}}$ and $F \subseteq \mathbb{R}$ be such that $|F|<\kappa$. By definition of $d_{\mathfrak{c}}$ there is a $g \in \mathbb{R}^{\mathbb{R}}$ such that $|[f=g]|<\mathfrak{c}$ for all $f \in F$. Let $g_{1}=-g$. Now $\left|\left(f+g_{1}\right)^{-1}(0)\right|<\mathfrak{c}$ for all $f \in F$. Thus, $f+g_{1} \in \neg$ Dar $(\mathfrak{c})$ for all $f \in F$. Since $F$ was an arbitrary set of cardinality less than $\kappa$, we have $\kappa<\mathrm{A}(\neg \operatorname{Dar}(\mathfrak{c}))$. It follows that $d_{\mathfrak{c}} \leq \mathrm{A}(\neg \operatorname{Dar}(\mathfrak{c}))$.

We now prepare to prove that $\mathrm{A}(\neg \mathrm{AC}) \leq d_{\mathfrak{c}}^{*}$. 
Lemma 28. Let $\left\{P_{\alpha}: \alpha<\mathfrak{c}\right\}$ be a partition of $\mathfrak{c}$ into $\mathfrak{c}$-many sets of cardinality $\mathfrak{c}$ and let

$$
\begin{aligned}
\kappa & =\min \left\{|F|: F \subseteq \mathfrak{c}^{\mathfrak{c}}\right. \\
& \left.\& \quad\left(\forall G \in\left[\mathfrak{c}^{\mathfrak{c}}\right]^{\mathfrak{c}}\right)(\exists f \in F)(\forall g \in G)(\forall \alpha<\mathfrak{c})\left(\left|P_{\alpha} \cap[f=g]\right|=\mathfrak{c}\right)\right\}
\end{aligned}
$$

Then $\kappa=d_{\mathfrak{c}}^{*}$.

Proof. We first show that $\kappa \leq d_{\mathfrak{c}}^{*}$. Let $F \subseteq \mathfrak{c}^{\mathfrak{c}}$ witness the definition of $d_{\mathfrak{c}}^{*}$, i.e. $|F|=d_{\mathfrak{c}}^{*}$ and

$$
\left(\forall G \in\left[\mathfrak{c}^{\mathfrak{c}}\right]^{\mathfrak{c}}\right)(\exists f \in F)(\forall g \in G)(|[f=g]|=\mathfrak{c}) .
$$

It is enough to find a family $F^{*} \subseteq \mathfrak{c}^{\mathfrak{c}}$ such that $\left|F^{*}\right|=|F|$ and,

$$
\left(\forall G \in\left[\mathfrak{c}^{\mathfrak{c}}\right]^{\mathfrak{c}}\right)\left(\exists f^{*} \in F^{*}\right)(\forall g \in G)(\forall \alpha<\mathfrak{c})\left(\left|P_{\alpha} \cap\left[f^{*}=g\right]\right|=\mathfrak{c}\right) .
$$

For every $\alpha<\mathfrak{c}$ enumerate $P_{\alpha}$ as $\left\{x_{\langle\alpha, \beta\rangle}: \beta<\mathfrak{c}\right\}$. For each $f \in F$ let $f^{*} \in \mathfrak{c}^{\mathfrak{c}}$ be given by $f^{*}\left(x_{\langle\alpha, \beta\rangle}\right)=f(\beta)$ for all $\langle\alpha, \beta\rangle \in \mathfrak{c}^{2}$. Clearly $\left|F^{*}\right|=|F|$. We show that $F^{*}$ satisfies (15). Let $G \in\left[\mathfrak{c}^{\mathfrak{c}}\right]^{\mathfrak{c}}$ be arbitrary. Enumerate $G$ by $\left\{g_{\xi}: \xi<\mathfrak{c}\right\}$. For each $\langle\alpha, \xi\rangle \in \mathfrak{c}^{2}$ define $g_{\langle\alpha, \xi\rangle} \in \mathfrak{c}^{\mathfrak{c}}$ so that $g_{\langle\alpha, \xi\rangle}(\beta)=$ $g_{\xi}\left(x_{\langle\alpha, \beta\rangle}\right)$ for all $\beta<\mathfrak{c}$. By $(14)$ there exists $f \in F$ such that $\left|\left[g_{\langle\alpha, \xi\rangle}=f\right]\right|=\mathfrak{c}$ for all $\langle\alpha, \xi\rangle \in \mathfrak{c}^{2}$. Fix $\langle\alpha, \xi\rangle \in \mathfrak{c}^{2}$. Then $\left|\left[g_{\langle\alpha, \xi\rangle}=f\right]\right|=\mathfrak{c}$ and for every $\beta \in\left[g_{\langle\alpha, \xi\rangle}=f\right]$,

$$
f^{*}\left(x_{\langle\alpha, \beta\rangle}\right)=f(\beta)=g_{\langle\alpha, \xi\rangle}(\beta)=g_{\xi}\left(x_{\langle\alpha, \beta\rangle}\right) .
$$

Thus, $\left|P_{\alpha} \cap\left[f^{*}=g_{\xi}\right]\right|=\mathfrak{c}$. Since $\langle\alpha, \xi\rangle \in \mathfrak{c}^{2}$ was arbitrary, we conclude that $F^{*}$ satisfies (15). So $\kappa \leq d_{\mathfrak{c}}^{*}$.

We now show the other inequality. Let $F \subseteq \mathfrak{c}^{\mathfrak{c}}$ witness the definition of $\kappa$, i.e., $F$ satisfies (15). It is enough to show that $F$ satisfies (14). Let $G \in\left[\mathfrak{c}^{\mathfrak{c}}\right]^{\mathfrak{c}}$ be arbitrary. Let $\left\{g_{\alpha}: \alpha<\mathfrak{c}\right\}$ be an enumeration of $G$. Pick $f \in F$ so that $\left|P_{\alpha} \bigcap[f=g]\right|=\mathfrak{c}$ for every $\alpha<\mathfrak{c}$. Clearly, we have $|[f=g]|=\mathfrak{c}$ for all $g \in G$. Thus, $F$ satisfies (14). The proof is complete.

We will need a fact about almost continuous functions which may be found in [5].

Proposition 29. (Natkaniec [5]) If $f \in \mathbb{R}^{\mathbb{R}}$ has non-empty intersection with every upper semi-continuous function u defined on a non-degenerate interval, then $f \in \mathrm{AC}$. 
Proof of $\mathrm{A}(\neg \mathrm{AC}) \leq d_{\mathfrak{c}}^{*}$. Let $\kappa=d_{\mathfrak{c}}^{*}$ and $\left\{P_{\alpha}: \alpha<\mathfrak{c}\right\}$ be a partition of $\mathbb{R}$ into $\mathfrak{c}$-many sets of cardinality $\mathfrak{c}$ so that for every open interval $I$ there is an $\alpha<\mathfrak{c}$ such that $P_{\alpha} \subseteq I$. By Lemma 28 there is an $F \in\left[\mathbb{R}^{\mathbb{R}}\right]^{\kappa}$ such that

$$
\left(\forall G \in\left[\mathbb{R}^{\mathbb{R}}\right]^{\mathfrak{c}}\right)(\exists f \in F)(\forall g \in G)(\forall \alpha<\mathfrak{c})\left(\left|P_{\alpha} \cap[f=g]\right|=\mathfrak{c}\right) .
$$

It is enough to show that

$$
\left(\forall g \in \mathbb{R}^{\mathbb{R}}\right)(\exists f \in F)(f+g \in \mathrm{AC}) .
$$

Let $U$ denote the family of upper semi-continuous functions in $\mathbb{R}^{\mathbb{R}}$ defined on a non-degenerate interval. We extend the domain of any partial function $u \in U$ to all of $\mathbb{R}$ in an arbitrary manner. Note that $|U|=\mathfrak{c}$. Let $g \in \mathbb{R}^{\mathbb{R}}$ be arbitrary and put $G=\{u-g: u \in U\}$. By (16) there is an $f \in F$ such that for every $\alpha \in \mathfrak{c}$ and $u \in U$ we have $\left|P_{\alpha} \bigcap[f=u-g]\right|=\mathfrak{c}$. So, $[f+g=u]$ is dense for each $u \in U$ by our choice of $\left\{P_{\alpha}: \alpha<\mathfrak{c}\right\}$. Thus, by Proposition 29, $f+g \in$ AC. Therefore, $F$ satisfies (17) which completes the proof.

Proof OF Theorem 8. We have proved $d_{\mathfrak{c}} \leq \mathrm{A}(\neg$ Dar $)$ and $\mathrm{A}(\neg \mathrm{AC}) \leq d_{\mathfrak{c}}^{*}$ while, by containment, we have $\mathrm{A}(\neg \mathrm{Dar}) \leq \mathrm{A}(\neg \mathrm{Con}) \leq \mathrm{A}(\neg \mathrm{AC})$.

\section{The family SZ}

To prove Theorem 9 we quote a theorem about SZ functions which may be found in [6].

Proposition 30. (Sierpiński, Zygmund [6]) $f \in \mathbb{R}^{\mathbb{R}}$ is in SZ if and only if $|[f=h]|<\mathfrak{c}$ for every continuous function defined on a $G_{\delta}$ set of cardinality $\mathfrak{c}$.

Proof of Theorem 9. We first show that $\mathrm{A}(\neg \mathrm{SZ}) \leq e_{\mathfrak{c}}^{*}$. Let $H$ stand for the family of all functions $h \in \mathbb{R}^{\mathbb{R}}$ such that $h$ is continuous on a $G_{\delta}$ set of cardinality $\mathfrak{c}$ and equal to zero elsewhere. Note that $|H|=\mathfrak{c}$. Let $F \subseteq \mathbb{R}^{\mathbb{R}}$ witness the definition of $e_{\mathfrak{c}}^{*}$, i.e., $|F|=e^{*}$ and

$$
\left(\forall G \in\left[\mathbb{R}^{\mathbb{R}}\right]^{\mathfrak{c}}\right)(\exists f \in F)(\forall g \in G)(|[f=g]|<\mathfrak{c}) .
$$

It is enough to show that $F$ satisfies

$$
(\forall g \in G)(\exists f \in F)(f+g \in \mathrm{SZ}) .
$$

Let $g \in \mathbb{R}^{\mathbb{R}}$ be arbitrary. Let $G=\{h-g: h \in H\}$. By (18), there exists an $f \in F$ such that $|[f=h-g]|<\mathfrak{c}$ for each $h \in H$. So, $|[f+g=h]|<\mathfrak{c}$ for 
each $h \in H$. Thus, by Proposition 30, $f+g \in \mathrm{SZ}$ and $F$ satisfies (19). So, $\mathrm{A}(\neg \mathrm{SZ}) \leq e_{\mathfrak{c}}^{*}$.

Next we show that $e_{\mathfrak{c}} \leq \mathrm{A}(\neg \mathrm{SZ})$. Let $F \subseteq \mathbb{R}^{\mathbb{R}}$ witness the definition of $\mathrm{A}(\neg \mathrm{SZ})$, i.e., $|F|=\mathrm{A}(\neg \mathrm{SZ})$ and $F$ satisfies (19). It is enough to show that $F$ satisfies

$$
\left(\forall g \in \mathbb{R}^{\mathbb{R}}\right)(\exists f \in F)(\mid[f=g]<\mathfrak{c}) .
$$

Let $g \in \mathbb{R}^{\mathbb{R}}$ be arbitrary. By (19) there is an $f \in F$ such that $-g+f \in$ SZ. Then $\left|\left[-g+f=\chi_{\emptyset}\right]\right|<\mathfrak{c}$, so $|[f+g]|<\mathfrak{c}$. Thus, $F$ satisfies $(20)$.

\section{Combinatorics}

We now prove Theorems 10 and 11.

Proof of Theorem 10. Let $W=\bigcup\left\{\mathbb{R}^{\alpha}: \alpha<\mathfrak{c}\right\}$. Note that $|W|=\mathfrak{c}$ by our assumption that $\left|[\mathfrak{c}]^{<\mathfrak{c}}\right|=\mathfrak{c}$. Let $V=\{\langle\alpha, \xi\rangle: \xi \leq \alpha<\mathfrak{c}\}$. We will use these sets throughout the proofs of $d_{\mathfrak{c}}=d_{\mathfrak{c}}^{*}$ and $e_{\mathfrak{c}}=e_{\mathfrak{c}}^{*}$.

We prove $d_{\mathfrak{c}}=d_{\mathfrak{c}}^{*}$. Since the inequality $d_{\mathfrak{c}} \leq d_{\mathfrak{c}}^{*}$ is obvious, we prove only that $d_{\mathfrak{c}}^{*} \leq d_{\mathfrak{c}}$. Let $F \subseteq W^{\mathfrak{c}}$ witness the definition of $d_{\mathfrak{c}}$, i.e., $|F|=d_{\mathfrak{c}}$ and

$$
\left(\forall g \in W^{\mathfrak{c}}\right)(\exists f \in F)(|[f=g]|=\mathfrak{c}) .
$$

It is enough to find a family $F^{*} \subseteq \mathfrak{c}^{V}$ of cardinality less than or equal to $|F|$ witnessing the definition of $d_{\mathfrak{c}}^{*}$, i.e.,

$$
\left(\forall G \in\left[\mathfrak{c}^{V}\right]^{\mathfrak{c}}\right)\left(\exists f \in F^{*}\right)(\forall g \in G)(|[f=g]|=\mathfrak{c}) .
$$

For each $f \in F$ let $f^{*} \in \mathfrak{c}^{V}$ be such that $f^{*}(\alpha, \beta)=f(\alpha)(\beta)$ if $\beta \in$ $\operatorname{dom}(f(\alpha))$ and zero otherwise. Let $F^{*}=\left\{f^{*}: f \in F\right\}$, note $\left|F^{*}\right| \leq|F|$. We show that $F^{*}$ satisfies (22) which will complete the proof. Let $G \in\left[\mathfrak{c}^{V}\right]^{\mathfrak{c}}$ be arbitrary and let $\left\{g_{\alpha}: \alpha<\mathfrak{c}\right\}$ be an enumeration of $G$. Define $h \in W^{\mathfrak{c}}$ so that

$$
h(\alpha) \in \mathfrak{c}^{\alpha} \text { and } h(\alpha)(\beta)=g_{\beta}(\alpha, \beta) \text { for all } \beta<\alpha .
$$

By (21), there exists an $f \in F$ such that $|[f=h]|=\mathfrak{c}$. We claim that $\left|\left[f^{*}=g_{\alpha}\right]\right|=\mathfrak{c}$ for all $\alpha<\mathfrak{c}$. Fix $\alpha<\mathfrak{c}$. Since $|[f=h]|=\mathfrak{c}$, the relations

$$
\alpha<\beta<\mathfrak{c} \text { and } f(\beta)=h(\beta)
$$

are satisfied by $\mathfrak{c}$-many $\beta<\mathfrak{c}$. Consider $\beta$ satisfying (24). By $(23), h(\beta) \in \mathfrak{c}^{\beta}$. So,

$$
f^{*}(\beta, \alpha)=f(\beta)(\alpha)=h(\beta)(\alpha)=g_{\alpha}(\beta, \alpha) .
$$


We conclude that $\mid\left[f^{*}=g_{\alpha} \mid=\mathfrak{c}\right.$. Thus, $F^{*}$ satisfies (22), completing the proof of $d_{\mathfrak{c}}=d_{\mathfrak{c}}^{*}$.

We prove $e_{\mathfrak{c}}=e_{\mathfrak{c}}^{*}$. The inequality $e_{\mathfrak{c}} \leq e_{\mathfrak{c}}^{*}$ is obvious. To prove $e_{\mathfrak{c}}^{*} \leq e_{\mathfrak{c}}$ let $F \subseteq \mathfrak{c}^{V}$ witness the definition of $e_{\mathfrak{c}}$, i.e., $|F|=e_{\mathfrak{c}}$ and

$$
\left(\forall g \in \mathfrak{c}^{V}\right)(\exists f \in F)(|[f=g]|<\mathfrak{c}) .
$$

It is enough to find a family $F^{*} \subset W^{\mathfrak{c}}$ of cardinality less than or equal to $|F|$ witnessing $e_{\mathfrak{c}}^{*}$, i.e.,

$$
\left(\forall G \in\left[W^{\mathfrak{c}}\right]^{\mathfrak{c}}\right)\left(\exists f^{*} \in F^{*}\right)(\forall g \in G)\left(\left|\left[f^{*}=g\right]\right|<\mathfrak{c}\right) .
$$

For each $f \in F$ let $f^{*} \in W^{\mathfrak{c}}$ be such that $f^{*}(\alpha) \in \mathfrak{c}^{\alpha}$ for every $\alpha<\mathfrak{c}$ and let $f^{*}(\alpha)(\beta)=f(\alpha, \beta)$ for every $\beta<\alpha$. Let $F^{*}=\left\{f^{*}: f \in F\right\}$ and note that $\left|F^{*}\right| \leq|F|$. We show that $F^{*}$ satisfies (26). Let $G \in\left[W^{\mathfrak{c}}\right]^{\mathfrak{c}}$ and let $\left\{g_{\alpha}: \alpha<\mathfrak{c}\right\}$ enumerate $G$. We find an $f^{*} \in F^{*}$ such that

$$
\left|\left[f^{*}=g_{\alpha}\right]\right|<\mathfrak{c} \text { for every } \alpha<\mathfrak{c} .
$$

Define $g \in \mathfrak{c}^{V}$ by $g(\beta, \alpha)=g_{\alpha}(\beta)(\alpha)$ if $\alpha \in \operatorname{dom}\left(g_{\alpha}(\beta)\right)$ and zero otherwise. By (25) there is some $f \in F$ such that $|f \cap g|<\mathfrak{c}$. We claim that $f^{*}$ satisfies (27), which will complete the proof. Fix $\alpha<\mathfrak{c}$. For any $\alpha<\beta<\mathfrak{c} f^{*}(\beta)=g_{\alpha}(\beta)$ implies that $f^{*}(\beta)(\gamma)=f(\beta, \gamma)=g_{\alpha}(\beta)(\gamma)$ for every $\gamma<\beta$. In particular, $f(\beta, \alpha)=g_{\alpha}(\beta)(\alpha)$ so $f(\beta, \alpha)=g(\beta, \alpha)$. Since $|[f=g]|<\mathfrak{c}$, there must be less than $\mathfrak{c}$ many $\beta>\alpha$ such that $f^{*}(\beta)=g_{\alpha}(\beta)$. Thus, $\left|\left[f^{*}=g_{\alpha}\right]\right|<\mathfrak{c}$. Since $\alpha<\mathfrak{c}$ was arbitrary, $f^{*}$ satisfies (27) completing the proof.

To prove Theorem 11 we will need three more lemmas and define two more cardinals.

$$
\begin{aligned}
& D_{\kappa}=\min \left\{|F|: F \subseteq \kappa^{\kappa} \&\left(\forall g \in \kappa^{\kappa}\right)(\exists f \in F)(|[f \leq g]|<\kappa)\right\}, \\
& b_{\kappa}=\min \left\{|F|: F \subseteq \kappa^{\kappa} \&\left(\forall g \in \kappa^{\kappa}\right)(\exists f \in F)(|[g \leq f]|=\kappa)\right\} .
\end{aligned}
$$

The numbers $b_{\mathfrak{c}}$ and $D_{\mathfrak{c}}$ are analogs of the bounding number $b=b_{\omega}$ and the dominating number $D=D_{\omega}$.

Lemma 31. If $\mathfrak{c}=\lambda^{+}$, then for every $f \in \mathfrak{c}^{\mathfrak{c}}$ the set $\left\{\langle\alpha, \beta\rangle \in \mathfrak{c}^{2}: \beta \leq f(\alpha)\right\}$ is the union of $\lambda$-many functions in $\mathfrak{c}^{\mathfrak{c}}$.

Proof. Let $f \in \mathfrak{c}^{\mathfrak{c}}$ be arbitrary. We find a family $\left\{f_{\zeta} \in \mathfrak{c}^{\mathfrak{c}}: \zeta<\lambda\right\}$ such that

$$
\left\{\langle\alpha, \beta\rangle \in \mathfrak{c}^{2}: \beta \leq f(\alpha)\right\}=\bigcup\left\{f_{\zeta}: \zeta<\lambda\right\}
$$


For each $\alpha<\mathfrak{c}$ let $\left\{\gamma_{\langle\alpha, \zeta\rangle}: \zeta<\lambda\right\}$ be an enumeration of $f(\alpha)+1$. For each $\zeta<\lambda$ define $f_{\zeta} \in \mathfrak{c}^{\mathfrak{c}}$ so that $f_{\zeta}(\alpha)=\gamma_{\langle\alpha, \zeta\rangle}$. We show that $\left\{f_{\zeta}: \zeta<\lambda\right\}$ satisfies (28). Fix $\alpha<\mathfrak{c}$. Then for any $\beta<\mathfrak{c}$ we have

$$
\beta \leq f(\alpha) \Leftrightarrow(\exists \zeta<\lambda)\left(\gamma_{\langle\alpha, \zeta\rangle}=\beta\right) \Leftrightarrow(\exists \zeta<\lambda)\left(f_{\zeta}(\alpha)=\beta\right) .
$$

Thus, (28) is satisfied.

Lemma 32. If $\operatorname{cf}(\mathfrak{c})=\mathfrak{c}$, then $b_{\mathfrak{c}}>\mathfrak{c}$.

Proof. Let $F \in\left[\mathfrak{c}^{\mathfrak{c}}\right]^{\mathfrak{c}}$ be arbitrary. It is enough to find $g \in \mathfrak{c}^{\mathfrak{c}}$ such that $|[g \leq f]|<\mathfrak{c}$ for every $f \in F$. Let $\left\{f_{\alpha}: \alpha \in \mathfrak{c}\right\}$ be an enumeration of $F$. Define $g \in \mathfrak{c}^{\mathfrak{c}}$ so that $g(\alpha)>f_{\beta}(\alpha)$ for all $\beta \leq \alpha$. We may make such a choice since $\operatorname{cf}(\mathfrak{c})=\mathfrak{c}$. To see that $g$ is as desired let $\alpha<\mathfrak{c}$ be arbitrary. Then $g(\beta)>f_{\alpha}$ for all $\beta>\alpha$.

The first part of the next lemma is due to Ciesielski [1].

Lemma 33. If $\mathfrak{c}=\lambda^{+}$, then $b_{\mathfrak{c}}=d_{\mathfrak{c}}$, and $D_{\mathfrak{c}}=e_{\mathfrak{c}}^{*}$.

Proof. We prove $b_{\mathfrak{c}}=d_{\mathfrak{c}}$. To show that $d_{\mathfrak{c}} \leq b_{\mathfrak{c}}$ let $F \subseteq \mathfrak{c}^{\mathfrak{c}}$ witness the definition of $b_{\mathfrak{c}}$, i.e., $|F|=b_{\mathfrak{c}}$ and

$$
\left(\forall g \in \mathfrak{c}^{\mathfrak{c}}\right)(\exists f \in F)(|[g \leq f]|=\mathfrak{c}) .
$$

It is enough to find $F^{*} \subseteq \mathfrak{c}^{\mathfrak{c}}$ such that $|F|=\left|F^{*}\right|$ and

$$
\left(\forall g \in \mathfrak{c}^{\mathfrak{c}}\right)\left(\exists f^{*} \in F^{*}\right)(|[f=g]|=\mathfrak{c}) .
$$

For each $f \in F$ let $\left\{f_{\zeta}: \zeta<\lambda\right\}$ be as in Lemma 31 and let $F^{*}=\bigcup\left\{\left\{f_{\zeta}: \zeta<\right.\right.$ $\lambda\}: f \in F\}$. By Lemma 32, $|F|=\left|F^{*}\right|$. We show that $F^{*}$ satisfies (30). Let $g \in \mathfrak{c}^{\mathfrak{c}}$ be arbitrary. By (29), there is an $f \in F$ such that $|[g \leq f]|=\mathfrak{c}$. Since $|g \bigcap\{\langle\alpha, \beta\rangle: \beta \leq f(\alpha)\}|=\mathfrak{c}$ and $\{\langle\alpha, \beta\rangle: \beta \leq f(\alpha)\}=\bigcup\left\{f_{\zeta}: \zeta<\lambda\right\}$, there is some $\zeta<\lambda$ such that $\left|\left[f_{\zeta}=g\right]=\right| f_{\zeta} \cap g \mid=\mathfrak{c}$. Thus, $F^{*}$ satisfies (30). So $d_{\mathfrak{c}} \leq b_{\mathfrak{c}}$. To see the other inequality notice that any family $F$ satisfying (30) also satisfies (29).

We prove $D_{\mathfrak{c}}=e_{\mathfrak{c}}^{*}$. To show that $D_{\mathfrak{c}} \leq e_{\mathfrak{c}}^{*}$ let $F \subseteq \mathfrak{c}^{\mathfrak{c}}$ witnesses the definition of $e_{\mathfrak{c}}^{*}$, i.e., $|F|=e_{\mathfrak{c}}^{*}$ and

$$
\left(\forall G \in\left[\mathfrak{c}^{\mathfrak{c}}\right]^{\mathfrak{c}}\right)(\exists f \in F)(\forall g \in G)(|[f=g]|<\mathfrak{c}) .
$$

It is enough to find $F^{*} \subseteq \mathfrak{c}^{\mathfrak{c}}$ such that $|F|=\left|F^{*}\right|$ and

$$
\left(\forall g \in \mathfrak{c}^{\mathfrak{c}}\right)(\exists f \in F)(|[f \leq g]|<\mathfrak{c}) .
$$


We show that $F$ itself satisfies (32). Let $g \in \mathfrak{c}^{\mathfrak{c}}$ be arbitrary. Let $\left\{g_{\zeta}: \zeta<\lambda\right\}$ be as in Lemma 31. By (31) there exists an $f \in F$ such that $\left|\left[f=g_{\zeta}\right]\right|<\mathfrak{c}$ for every $\zeta<\lambda$. Since, $\operatorname{cf}(\mathfrak{c})=\mathfrak{c}$ and $\{\langle\alpha, \beta\rangle: \beta \leq g(\alpha)\}=\bigcup\left\{g_{\zeta}: \zeta<\lambda\right\}$, we have $|[f \leq g]|<\mathfrak{c}$. Thus, $F$ satisfies (32). So, $D_{\mathfrak{c}} \leq e_{\mathfrak{c}}^{*}$. We show that $e_{\mathfrak{c}}^{*} \leq D_{\mathfrak{c}}$. It is enough to prove that any family $F$ satisfying (32) also satisfies (31). Suppose $F$ satisfies (32). Let $G \in\left[\mathfrak{c}^{\mathfrak{c}}\right]^{\mathfrak{c}}$ be arbitrary and $\left\{g_{\alpha}: \alpha<\mathfrak{c}\right\}$ be an enumeration of $G$. By Lemma 32 there is an $h \in \mathfrak{c}^{\mathfrak{c}}$ such that $\left|\left[h \leq g_{\alpha}\right]\right|<\mathfrak{c}$ for each $\alpha<\mathfrak{c}$. By (32) there exists an $f \in F$ such that $|[f \leq h]|<\mathfrak{c}$. It follows that $\left|\left[f \leq g_{\alpha}\right]\right|<\mathfrak{c}$ for all $\alpha<\mathfrak{c}$. In particular, $|[f=g]|<\mathfrak{c}$ for every $g \in G$, so $F$ satisfies (31). Thus, $e_{\mathfrak{c}}^{*}<D_{\mathfrak{c}}$.

Proof of Theorem 11. Clearly $b_{\mathfrak{c}} \leq D_{\mathfrak{c}}$. By Lemma 33 we have $d_{\mathfrak{c}} \leq e_{\mathfrak{c}}^{*}$. By the second part of Theorem 10, $e_{\mathfrak{c}}^{*}=e_{\mathfrak{c}}$. Thus, $d_{\mathfrak{c}} \leq e_{\mathfrak{c}}$.

\section{References}

[1] K. Ciesielski, Private communication.

[2] K. Ciesielski and A.W. Miller, Cardinal invariants concerning functions, whose sum is almost continuous, Real Anal. Exchange 20 (1994/95), 657672 .

[3] K. Ciesielski and T. Natkaniec, Algebraic properties of the class of Sierpiński-Zygmund functions, Topology Appl., to appear.

[4] K. Ciesielski and I. Recław, Cardinal invariants concerning extendable and peripherally continuous functions, Real Anal. Exchange 21 (1995/96), $459-472$.

[5] T. Natkaniec, Almost continuity, Real Analysis Exchange 17 (1991-92), $462-520$.

[6] W. Sierpiński and A. Zygmund, Sur une fonction qui est discontinue sur tout ensemble de puissance du continu, Fund. Math. 4 (1923), 316-318. 